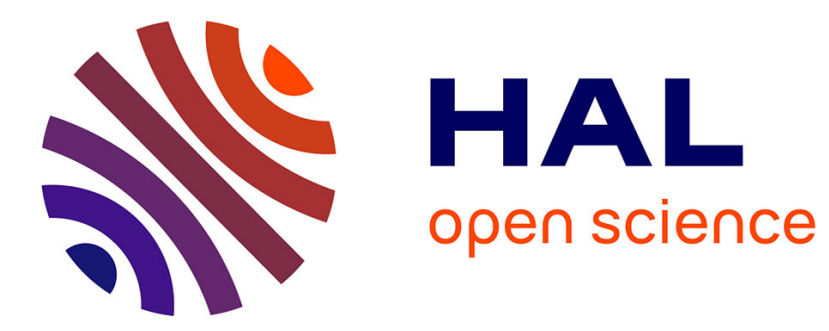

\title{
Spectroscopie du chrome très ionisé : niveaux d'énergie de la configuration 2p531 de Cr XV
}

\author{
M.C. Buchet-Poulizac, J.P. Buchet, S. Martin
}

\section{To cite this version:}

M.C. Buchet-Poulizac, J.P. Buchet, S. Martin. Spectroscopie du chrome très ionisé: niveaux d'énergie de la configuration 2p531 de Cr XV. Journal de Physique, 1986, 47 (3), pp.407-416. 10.1051/jphys:01986004703040700 . jpa-00210220

\section{HAL Id: jpa-00210220 https://hal.science/jpa-00210220}

Submitted on 1 Jan 1986

HAL is a multi-disciplinary open access archive for the deposit and dissemination of scientific research documents, whether they are published or not. The documents may come from teaching and research institutions in France or abroad, or from public or private research centers.
L'archive ouverte pluridisciplinaire HAL, est destinée au dépôt et à la diffusion de documents scientifiques de niveau recherche, publiés ou non, émanant des établissements d'enseignement et de recherche français ou étrangers, des laboratoires publics ou privés. 
Classification

Physics Abstracts

$32.30 \mathrm{~J}-32.70 \mathrm{~F}$

\title{
Spectroscopie du chrome très ionisé : niveaux d'énergie de la configuration $2 p^{5} 31$ de $\mathrm{Cr} X \mathrm{~V}$
}

\author{
M. C. Buchet-Poulizac, J. P. Buchet et S. Martin \\ Laboratoire de Spectrométrie Ionique et Moléculaire $\left({ }^{*}\right)$, Université Lyon I, \\ Campus de La Doua, 69622 Villeurbanne Cedex, France
}

(Reçu le 19 juin 1985, accepté le 7 novembre 1985)

\begin{abstract}
Résumé. - La technique « beam-foil » a été utilisée pour produire les spectres du chrome très ionisé. L'étude dans l'ultraviolet lointain a permis en particulier l'analyse de $\mathrm{Cr} X \mathrm{XV}$. Les niveaux d'énergie de la configuration $2 \mathrm{p}^{5} 31$ ont été déterminés avec une précision de l'ordre de $150 \mathrm{~cm}^{-1}$. Les probabilités des deux transitions les plus intenses du spectre de $\mathrm{Cr} X V$ ont été déduites de la mesure des durées de vie de leurs niveaux supérieurs $2 \mathrm{p}^{5} 3 \mathrm{p}^{3} \mathrm{D}_{3}$ et $2 p^{5} 3 d{ }^{3} F_{4}$ et sont comparées aux résultats théoriques donnés dans la littérature.
\end{abstract}

\begin{abstract}
The « beam-foil " study in the far ultraviolet region has yielded spectra of highly ionized chromium. In particular, the spectrum of $\mathrm{Cr} X V$ has been analysed and energy levels of the $2 \mathrm{p}^{5} 31$ configuration have been determined with a mean accuracy of $150 \mathrm{~cm}^{-1}$. Lifetimes of the $2 p^{5} 3 p^{3} D_{3}$ and $2 p^{5} 3 d^{3} F_{4}$ levels have been measured and the transition probabilities deduced are compared with available theoretical data.
\end{abstract}

\section{Conditions expérimentales.}

L'injecteur du C.E.V. d'Orsay, ALICE, permet d'obtenir avec une intensité de l'ordre du $\mu \mathrm{A}$, des faisceaux d'ions métalliques $(\mathrm{Cr} \rightarrow \mathrm{Cu})$ d'énergie $1,16 \mathrm{MeV} / \mathrm{nu}$ cléon. Après la traversée d'une cible mince de carbone $\left(20 \mu \mathrm{g} / \mathrm{cm}^{2}\right)$, les états de charge moyens des ions excités correspondent aux séquences isoélectroniques $\mathrm{du}$ fluor, du néon et du sodium.

Nous avons poursuivi l'analyse dans l'ultraviolet lointain des ions isoélectroniques au néon $[1,2]$ en étudiant le spectre du chrome entre $80 \AA \AA$ et $480 \AA$. Nous avons utilisé un monochromateur à incidence rasante McPherson de 2,2 m, équipé d'un channeltron et d'un réseau gravé de 600 traits $/ \mathrm{mm}$. Pour l'angle d'incidence choisi $\left(82^{\circ}\right)$, le blaze du réseau était situé vers $250 \AA$.

Un premier spectre général a été enregistré avec des fentes de $90 \mu \mathrm{m}$ pour explorer tout le domaine spectral. Pour résoudre les nombreuses superpositions apparaissant dans ce spectre, il s'est avéré nécessaire d'améliorer la résolution spectrale. Compte tenu de l'élargissement Doppler dû à l'angle solide d'ouverture du spectromètre et affectant les raies émises par des ions animés d'une vitesse de l'ordre de $15 \times 10^{8} \mathrm{~cm} / \mathrm{s}$, nous avons fermé les fentes à $40 \mu \mathrm{m}$ et exploré en détail la

(*) L.A. $\mathbf{n}^{\circ} 171$. région $280-420 \AA$ où se situent les transitions 3-3 les plus intenses de $\mathrm{Cr} \mathrm{XV}$.

L'étalonnage du spectromètre avait été réalisé au préalable, en enregistrant des spectres d'hélium et de néon. Le gaz injecté sous faible pression dans l'enceinte d'expérience, était excité par une jauge à ionisation de type Penning. Les longueurs d'onde des raies émises par le faisceau d'ions ont été ultérieurement corrigées de l'effet Doppler.

\section{Longueurs d'onde.}

Les spectres enregistrés montrent principalement les raies de résonance du type $2 \mathrm{~s}^{2} 2 \mathrm{p}^{k}-2 \mathrm{~s} 2 \mathrm{p}^{k+1}$ de Cr XVI à Cr XIX, entre 100 et $170 \AA$ et qui apparaissent aussi sur nos spectres dans les deuxième et troisième ordres, les transitions 3-3 de Cr XIV et Cr XV, entre 280 et $480 \AA$, et les transitions hydrogénoïdes $\Delta n=1$ et 2 issues de niveaux $n$ élevés $(n \geqslant 5)$ pour Cr XIV à XIX. Ces dernières transitions, surtout celles issues de niveaux $n>7$, sont peu sensibles à l'effet de polarisation de coeur et leurs longueurs d'onde peuvent être prédites avec précision. Elles peuvent ainsi servir de référence et permettent de vérifier l'étalonnage. L'incertitude sur les mesures de longueurs d'onde est estimée à $0,03 \AA ̊$ en général, sauf quand les raies sont particulièrement faibles ou mal séparées. 
La figure 1 montre la région spectrale $320-480 \AA$, où sont situées les transitions 3-3 les plus intenses appartenant à la configuration $2 \mathrm{p}^{5} 31 \mathrm{de} \mathrm{Cr} \mathrm{XV}$. La figure 2 présente le spectre fait en fentes fines entre $285 \AA$ et $325 \AA$, où se trouvent les transitions $2 p^{5} 3 p-2 p^{5} 3 d$ de Cr XV.

Le tableau I donne les longueurs d'onde des raies de résonance de Cr XVI à Cr XIX et des transitions 3-3 de Cr XIV que nous avons observées. Il rappelle les valeurs expérimentales précédemment trouvées dans les plasmas de Tokamak $[3,4]$ ou autre plasma de laboratoire [5-8]. Enfin, il les compare aux tables de Fawcett $[9,10]$ et aux valeurs calculées par Edlén [11-13] dans ses analyses critiques des données expérimentales et théoriques pour les séquences isoélectroniques du sodium, du fluor, de l'oxygène

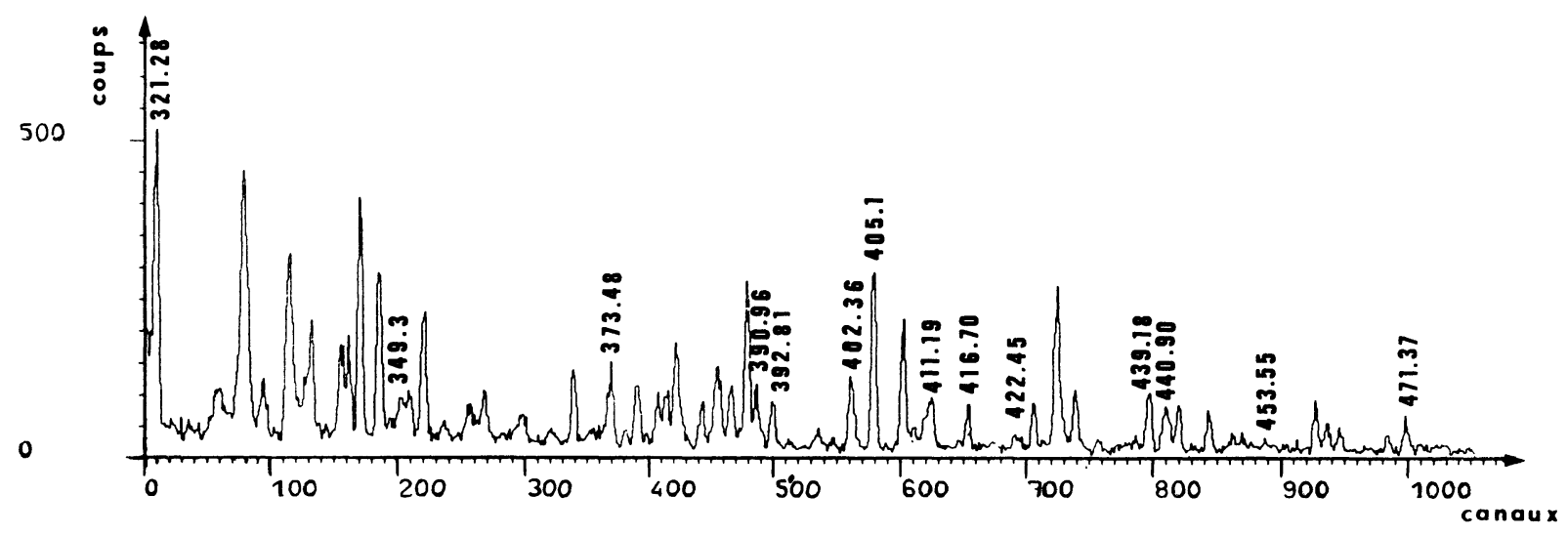

Fig. 1. - Spectre du chrome en fentes larges $(90 \mu \mathrm{m})$ entre $320 \AA$ et $480 \AA$. Les longueurs d'onde des raies que nous avons attribuées aux transitions $2 p^{5} 3 s-2 p^{5} 3 p$ et $2 p^{5} 3 p-2 p^{5} 3 d$ de $\mathrm{Cr} X V$ sont données en angström.

[Chromium spectrum recorded with $90 \mu \mathrm{m}$ slit width between $320 \AA$ and $480 \AA$. The wavelengths of the most intense transitions that we ascribed to $\mathrm{Cr} \mathrm{XV}$ are given in $\AA$.]

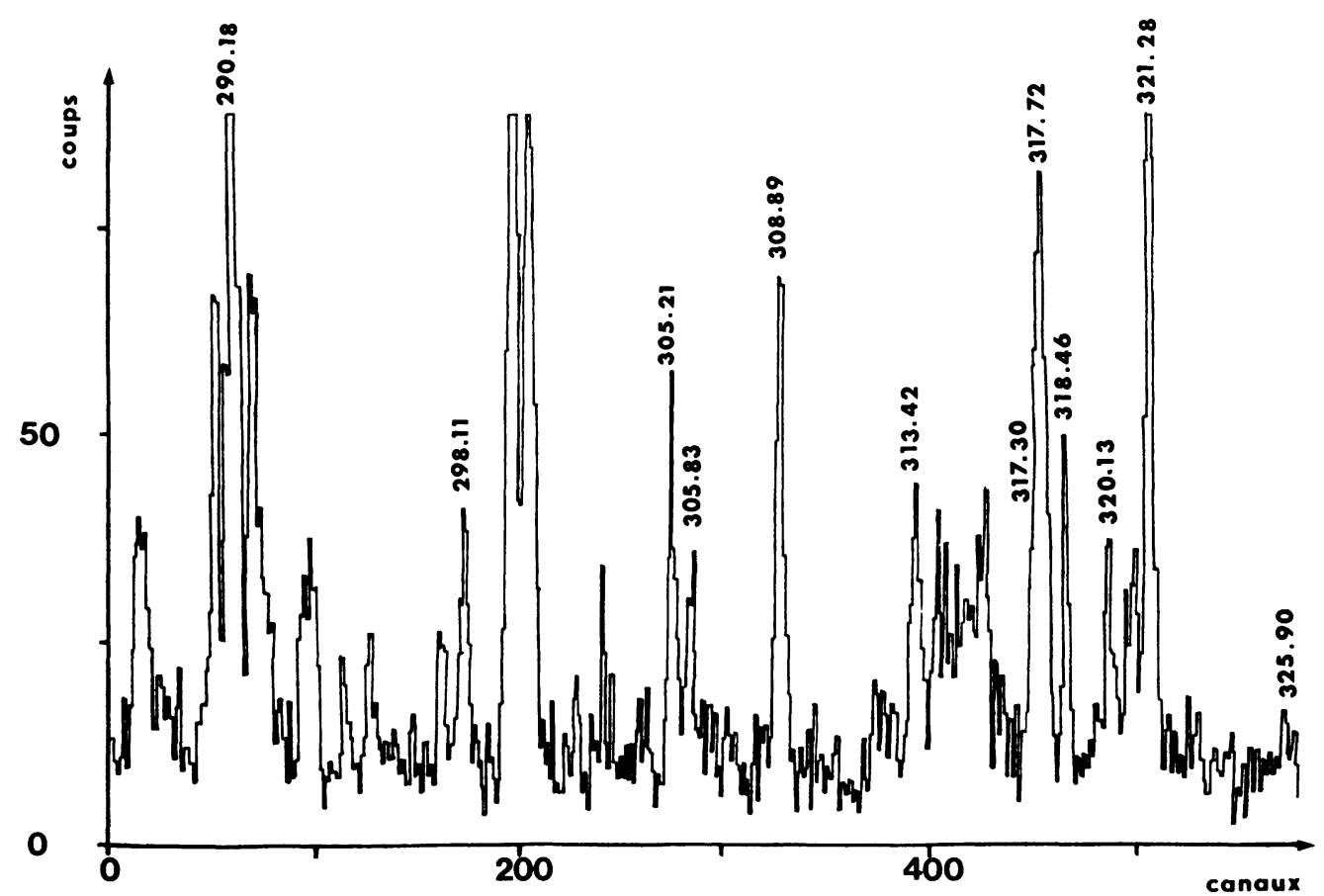

Fig. 2. - Spectre en fentes fines $(40 \mu \mathrm{m})$ entre $285 \AA$ et $325 \AA$. Les longueurs d'onde des transitions $2 \mathrm{p}^{5} 3 \mathrm{p}-2 \mathrm{p}^{5} 3 \mathrm{~d}$ de $\mathrm{Cr} \mathrm{XV}$ sont indiquées en angström.

[Partial spectrum recorded with $40 \mu \mathrm{m}$ slit width between $285 \AA$ and $325 \AA$. The wavelengths of the $2 p^{5} 3 p-2 p^{5} 3 d$ transitions of $\mathrm{Cr} \mathrm{XV}$ are given in $\AA$.] 
Tableau I. - Longueurs d'onde des raies de résonance du type $2 \mathrm{~s}^{2} 2 \mathrm{p}^{k}-2 \mathrm{~s} 2 \mathrm{p}^{k+1}$ de Cr XVI-XIX et des transitions 3-3 de Cr XIV.

[Wavelengths of the $2 \mathrm{~s}^{2} 2 \mathrm{p}^{k}-2 \mathrm{~s} 2 \mathrm{p}^{k+1}$ resonance lines of Cr XVI-XIX and of the 3-3 transitions of Cr XIV.]

\begin{tabular}{|c|c|c|c|c|}
\hline Transition & $\begin{array}{c}\text { Expérience }(\AA) \\
\text { nos résultats }\end{array}$ & Tokamak & Laser & Compilation $(\AA)$ \\
\hline 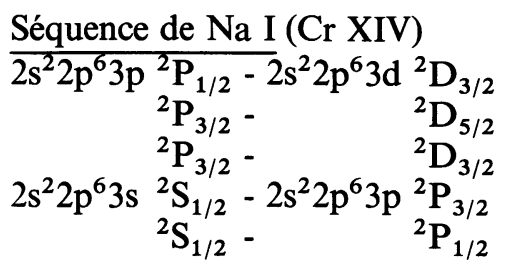 & $\begin{array}{l}289,75 \pm 0,05 \\
300,3(+\mathrm{CrXV}) \\
301,89 \pm 0,05 \\
389,82 \pm 0,05 \\
412,04 \pm 0,05\end{array}$ & $\begin{array}{l}389,864\left({ }^{a}\right) \\
412,051\left(^{(a)}\right.\end{array}$ & $\begin{array}{l}289,70\left(^{c}\right) \\
300,23\left(^{c}\right) \\
301,78\left(^{c}\right) \\
389,81\left(^{c}\right) \\
411,99\left(^{c}\right)\end{array}$ & $\begin{array}{l}289,753\left({ }^{\mathrm{i}}\right) \\
300,296\left(^{\mathrm{i}}\right) \\
301,831\left(^{\mathrm{i}}\right) \\
389,859\left(^{\mathrm{i}}\right) \\
412,045\left(^{\mathrm{i}}\right)\end{array}$ \\
\hline $\begin{array}{c}\text { Séquence de F I (Cr XVI) } \\
\frac{\mathrm{s}^{2} 2 \mathrm{p}^{5}{ }^{2} \mathrm{P}_{3 / 2}-2 \mathrm{~s} 2 \mathrm{p}^{6}{ }^{2} \mathrm{~S}_{1 / 2}}{{ }^{2} \mathrm{P}_{1 / 2}-} \\
{ }^{2} \mathrm{~S}_{1 / 2}\end{array}$ & $\begin{array}{l}106,64 \pm 0,03 \\
115,40 \pm 0,05\end{array}$ & $\begin{array}{l}106,64\left(^{(b)}\right. \\
115,38\left(^{b}\right)\end{array}$ & $\begin{array}{l}106,62\left({ }^{d}\right) \\
115,33(d)\end{array}$ & $\begin{array}{ll}106,600\left(^{(g)}\right. & 106,635\left(^{j}\right) \\
115,334\left({ }^{\mathbf{g}}\right) & 115,356\left(^{(j)}\right.\end{array}$ \\
\hline 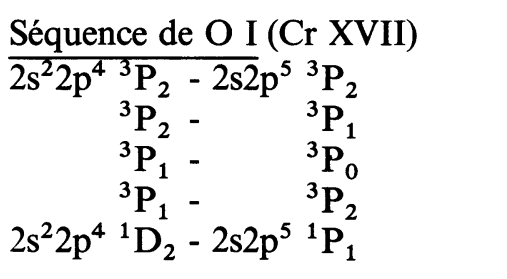 & $\begin{array}{l}122,97 \pm 0,03 \\
116,58 \pm 0,03 \\
120,89 \pm 0,03 \\
132,83 \pm 0,03 \\
101,91 \pm 0,05\end{array}$ & $\begin{array}{l}122,99\left(^{b}\right) \\
116,61\left(^{b}\right) \\
120,89\left(^{b}\right) \\
132,87\left(^{b}\right) \\
101,93\left(\left(^{b}\right)\right.\end{array}$ & $\begin{array}{l}122,93\left({ }^{d}\right) \\
116,57\left({ }^{d}\right) \\
120,87\left({ }^{d}\right) \\
132,76\left(^{d}\right) \\
101,93\left({ }^{d}\right)\end{array}$ & $\begin{array}{ll}122,91\left(^{\mathrm{h}}\right) & 122,972\left(^{\mathrm{j}}\right) \\
116,53\left(^{\mathrm{h}}\right) & 116,598\left(^{\mathrm{j}}\right) \\
120,84\left(^{\mathrm{h}}\right) & 120,897\left(^{\mathrm{j}}\right) \\
132,76\left(^{\mathrm{h}}\right) & 132,834\left(^{\mathrm{j}}\right) \\
101,91\left(^{\mathrm{h}}\right) & 101,929\left(^{\mathrm{j}}\right)\end{array}$ \\
\hline 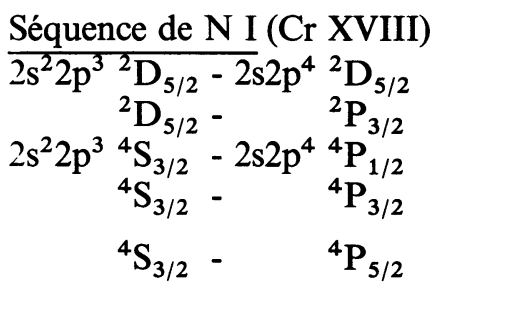 & $\begin{array}{l}128,13 \pm 0,03 \\
104,97 \pm 0,05 \\
136,63 \pm 0,03 \\
139,96 \pm 0,03 \\
149,86 \pm 0,03\end{array}$ & $\begin{array}{l}128,15\left(^{\mathrm{b}}\right) \\
104,98\left(^{\mathrm{b}}\right) \\
136,63\left(^{\mathrm{b}}\right) \\
139,98\left(^{\mathrm{b}}\right) \\
149,89\left(^{\mathrm{b}}\right)\end{array}$ & $\begin{array}{c}128,09\left({ }^{d}\right) \\
104,98\left(^{d}\right) \\
136,52\left({ }^{d}\right) \\
139,87\left(^{d}\right) \\
\left\{\begin{array}{l}149,66\left({ }^{d}\right) \\
149,80\left({ }^{\mathrm{e}}\right)\end{array}\right.\end{array}$ & 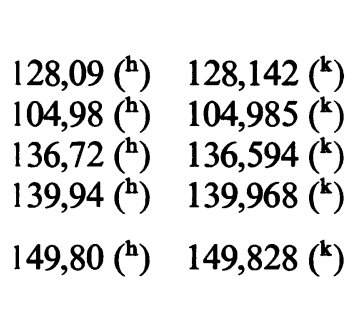 \\
\hline 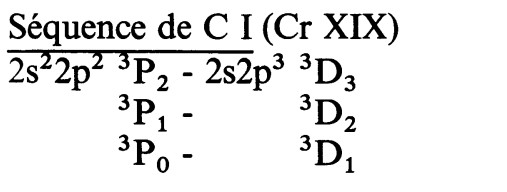 & $\begin{array}{l}165,49 \pm 0,03 \\
160,33 \pm 0,03 \\
148,69 \pm 0,03\end{array}$ & $\begin{array}{l}165,46\left(^{(b}\right) \\
160,34\left(^{b}\right)\end{array}$ & $\begin{array}{l}165,46\left({ }^{f}\right) \\
160,30\left(^{f}\right) \\
148,64\left(^{e}\right)\end{array}$ & $\begin{array}{l}165,47\left(\left(^{\mathbf{h}}\right)\right. \\
160,30\left(^{\mathbf{h}}\right) \\
148,47\left(^{\mathbf{h}}\right) \text { (prédite) }\end{array}$ \\
\hline
\end{tabular}
( $\left.{ }^{a}\right)$ Référence [3]
(e) Référence [7]
(i) Référence [11]
(b) Référence [4]
(f) Référence [8]
(j) Référence [12]
(c) Référence [5]
(8) Référence [10]
(k) Référence [13]
(d) Référence [6]
( $\left.{ }^{\mathrm{h}}\right)$ Référence [9]

et de l'azote. Nos mesures sont en excellent accord avec les calculs d'Edlén dans la limite de nos incertitudes.

Jupén et Litzén [14] avaient identifié dans un spectre du chrome émis dans un plasma produit par impact laser, six raies intenses de $\mathrm{Cr} X \mathrm{XV}$. Nous avons complété ce travail et identifié une vingtaine de transitions supplémentaires en nous basant sur des interpolations le long de la séquence du néon entre $\operatorname{Ar}$ IX [1], Ti XIII $[14,15]$ et Fe XVII [2] et sur les calculs de Bureeva et Safronova [16] (cf. pour plus de détails l'article cité en référence [2]).

Le tableau II donne les longueurs d'onde des raies attribuées à $\mathrm{Cr} \mathrm{XV}$ ainsi que leur identification.

Nous avons aussi indiqué les mélanges ou superpositions possibles. Quelques remarques plus détaillées sont cependant nécessaires :

- La raie située à $300,3 \AA$ a été attribuée à la transition $2 p^{6} 3 p^{2} P_{3 / 2}-2 p^{6} 3 d^{2} D_{5 / 2}$ de Cr XIV et à la transition $2 p^{5} 3 p^{3} S_{1}-2 p^{5} 3 d^{3} P_{1}$ de $C r X V$, l'intensité de la raie ne pouvant s'expliquer que par la superposition de ces deux transitions.

- Le pic centré sur $317,6 \AA$ sur nos spectres correspond au mélange des transitions $2 p^{5} 3 p{ }^{3} \mathrm{P}_{2}$ $2 p^{5} 3 d^{3} D_{3}$ et $2 p^{5} 3 p{ }^{1} D_{2}-2 p^{5} 3 d{ }^{1} F_{3}$. Les longueurs d'onde extraites de l'analyse du pic sont 317,3 $\pm 0,1 \AA$ et $317,72 \pm 0,05 \AA$ que l'on a attribuées respective- 
Tableau II. - Longueurs d'onde des transitions $2 \mathrm{p}^{5} 3 \mathrm{~s}-2 \mathrm{p}^{5} 3 \mathrm{p}$ et $2 \mathrm{p}^{5} 3 \mathrm{p}-2 \mathrm{p}^{5} 3 \mathrm{~d}$ de Cr XV.

[Wavelengths of the $2 p^{5} 3 s-2 p^{5} 3 p$ and $2 p^{5} 3 p-2 p^{5} 3 d$ transitions in Cr XV.]

\begin{tabular}{|c|c|c|c|}
\hline Transition & $\begin{array}{c}\text { Longueur d'onde } \\
(\AA) \\
\text { nos résultats }\end{array}$ & Laser $\left({ }^{a}\right)$ & Remarques \\
\hline 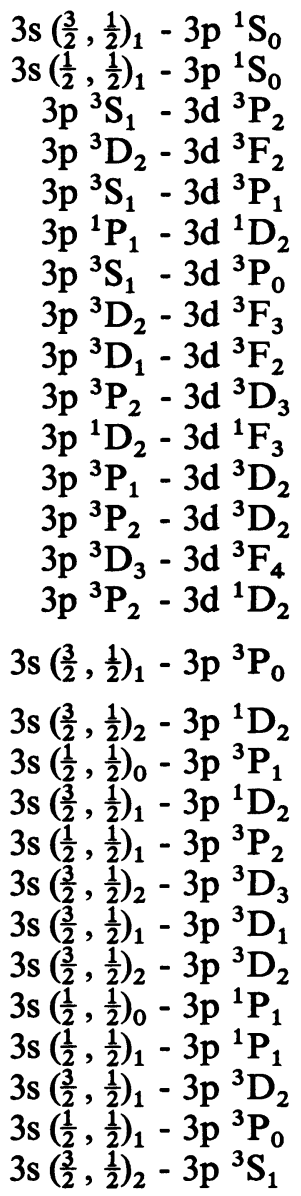 & $\begin{array}{l}237,7 \pm 0,1 \\
281,67 \pm 0,05 \\
290,18 \pm 0,03 \\
298,11 \pm 0,03 \\
300,30 \pm 0,03 \\
305,21 \pm 0,03 \\
305,83 \pm 0,03 \\
308,89 \pm 0,03 \\
313,42 \pm 0,03 \\
317,3 \pm 0,1 \\
317,72 \pm 0,05 \\
318,46 \pm 0,03 \\
320,13 \pm 0,03 \\
321,28 \pm 0,03 \\
325,9 \pm 0,1 \\
349,3 \pm 0,1 \\
373,48 \pm 0,03 \\
390,96 \pm 0,03 \\
392,81 \pm 0,03 \\
402,36 \pm 0,03 \\
405,11 \pm 0,05 \\
411,19 \pm 0,03 \\
416,70 \pm 0,05 \\
422,45 \pm 0,05 \\
439,15 \pm 0,05 \\
440,90 \pm 0,05 \\
453,55 \pm 0,05 \\
471,37 \pm 0,05\end{array}$ & $\begin{array}{l}308,861 \\
317,008 \\
317,711 \\
321,262\end{array}$ & $\begin{array}{l}+ \text { Cr XIV }(300,30) \\
+3^{e} \text { ordre Cr XVII }(101,91) \\
\text { raie mal séparée de } 317,72 \\
+\left\{\begin{array}{l}3^{\mathrm{e}} \text { ordre Cr XVII }(116,64) \\
7-9 \mathrm{Cr} \text { XVIII }(348,9)\end{array}\right) \\
+7-9 \mathrm{Cr} \text { XVII }(391,1) \\
+3 \mathrm{~s}\left(\frac{1}{2}, \frac{1}{2}\right)_{1}-3 \mathrm{p}^{3} \mathrm{P}_{1} \mathrm{Cr} \mathrm{XV} \\
+7-9 \mathrm{Cr} \text { XVI }(441,5)\end{array}$ \\
\hline
\end{tabular}

() Référence [14]

ment aux deux transitions, compte tenu des intensités relatives des deux composantes et des prédictions théoriques de Bureeva et Safronova [16].

- L'extrapolation des nombres d'onde, le long de la séquence isoélectronique, permet de prévoir les longueurs d'onde des transitions $2 \mathrm{p}^{5} 3 \mathrm{~s}(3 / 2,1 / 2)_{2}$ $2 p^{5} 3 p^{3} D_{3}$ et $2 p^{5} 3 s(1 / 2,1 / 2)_{1}-2 p^{5} 3 p^{3} P_{1}$ vers $405,1 \AA$. D'autre part, l'identification des raies situées à $390,96 \AA, 422,45 \AA$ et $439,15 \AA$ (cf. Tableau II), permet de prédire la longueur d'onde de la transition issue du niveau $2 p^{5} 3 p^{3} P_{1}$. Le calcul donne 405,22 \pm $0,15 \AA$. Sur l'enregistrement fait en fentes fines, la raie observée à $405,11 \AA$ semble effectivement plus large qu'une raie parfaitement pure. Cependant la comparaison des intensités des deux transitions dans la séquence montre que la transition issue du niveau $2 p^{5} 3 p^{3} D_{3}$ est beaucoup plus intense que celle issue du niveau $2 p^{5} 3 p^{3} P_{1}(\sim 13$ fois dans Fe XVII) aussi, bien que l'existence des deux transitions soit probable, nous identifions la raie $405,11 \AA$ à la transition $2 p^{5} 3 s(3 / 2,1 / 2)_{2}-2 p^{5} 3 p^{3} D_{3}$.

\section{Niveaux d'énergie.}

Les identifications ont permis de construire un schéma des niveaux d'énergie à partir des niveaux $2 \mathrm{p}^{5} 3 \mathrm{~s}$ $(3 / 2,1 / 2)_{1}$ et $2 p^{5} 3 s(1 / 2,1 / 2)_{1}$. Ces niveaux sont reliés au fondamental $2 \mathrm{p}^{6}{ }^{1} \mathrm{~S}_{0}$ par des raies de résonance situées dans le domaine des $\mathrm{X}$ et leurs énergies ont été tabulées par Sugar et Corliss [17]. Plus récemment, Klapisch et al. [18] ont observé dans un plasma de Tokamak, près des raies de résonance, la transition quadripolaire magnétique $\left(\mathbf{M}_{2}\right)$ ce qui permet de positionner le niveau $2 p^{5} 3 s(3 / 2,1 / 2)_{2}$. Dans un premier temps, nous nous sommes appliqués à mesurer les différences d'énergie entre les niveaux $2 \mathrm{p}^{5} 3 \mathrm{~s}$. 
Les calculs de Bureeva et Safronova [16] et Crance [19] permettent de prédire que les transitions issues des niveaux $2 p^{5} 3 p^{3} P_{0}$ et $2 p^{5} 3 p^{1} S_{0}$ et retombant sur les niveaux $2 \mathrm{p}^{5} 3 \mathrm{~s}$ de moment $j=1$, doivent être observables. Nous avons donc cherché les couples de raies correspondants, en se basant sur les variations le long de la séquence de $\mathrm{Ne}$ I. Des longueurs d'onde des raies à $349,3 \pm 0,1 \AA$ et $453,55 \pm 0,05 \AA$ que nous avons attribuées aux transitions issues du niveau $2 \mathrm{p}^{5} 3 \mathrm{p}{ }^{3} \mathrm{P}_{0}$, nous avons déduit une première valeur de l'écart $\Delta E$ entre les niveaux $2 \mathrm{p}^{5} 3 \mathrm{~s}$ de moment $j=1\left(65804 \pm 110 \mathrm{~cm}^{-1}\right)$ et la position du niveau $2 p^{5} 3 p^{3} P_{0}$. Ce même travail pour trouver les transitions issues du niveau $2 \mathrm{p}^{5} 3 \mathrm{p}{ }^{1} \mathrm{~S}_{0}$ était beaucoup plus imprécis car les résultats théoriques et expérimentaux sont plus dispersés tout le long de la séquence. Nous pouvions proposer deux couples de raies à 241,8 et $287,4 \AA$ ou à 237,7 et $281,67 \AA$. Nous avons préféré ce dernier couple de raies car il semble plus cohérent avec les observations faites dans le titane $[14,15]$. L'intervalle $\Delta E$ que nous pouvons déduire est égal à $65672 \pm 250 \mathrm{~cm}^{-1}$. Nos deux mesures sont très proches et en bon accord avec les valeurs données par Sugar et Corliss [17], néanmoins l'incertitude sur les mesures reste grande, les raies étant faibles ou partiellement mélangées.

Par contre, l'écart entre les niveaux $2 p^{5} 3 s(3 / 2,1 / 2)$ 1 et $2 \mathrm{p}^{5} 3 \mathrm{~s}(3 / 2,1 / 2)_{2}\left(13172 \mathrm{~cm}^{-1}\right)$ a été déterminé à partir de deux couples de raies intenses $(392,81$ $373,48 \AA$ et $416,70-440,90 \AA$ ) et nous pouvons affirmer que l'incertitude sur sa mesure est inférieure à $40 \mathrm{~cm}^{-1}$.

La plupart des autres niveaux sont connectés entre eux par au moins deux transitions situées dans une région spectrale peu perturbée par les deuxièmes ordres. Ceci nous a permis, d'une part de vérifier les identifications proposées, d'autre part de chiffrer lincertitude sur les énergies (en général inférieure à $100 \mathrm{~cm}^{-1}$ ).

Le tableau III donne les énergies des niveaux de $\mathrm{Cr} \mathrm{XV}$ que nous avons pu déduire de nos mesures avec leurs incertitudes ainsi que les données expérimentales $[17,18]$ ou théoriques les plus récentes sur ce sujet $[16,19]$. Les niveaux $2 \mathrm{p}^{5} 3 \mathrm{~s}$ sont dénommés selon le couplage $j j$ tandis que les niveaux des configurations $2 p^{5} 3 p$ et $2 p^{5} 3 d$ sont désignés selon la notation LS par continuité avec les analyses les plus récentes des éléments voisins de la séquence. Les désignations utilisées par Crance [19] ont cependant été rappelées entre parenthèses dans le tableau pour en faciliter la lecture. Le désaccord entre les énergies déduites de nos mesures et de celles de Kaplisch et al. [18] n'est qu'apparent, il ne représente qu'une différence inférieure à $3 / 1000$ d'angström sur la transition $2 \mathrm{p}^{6}{ }^{1} \mathrm{~S}_{0}-2 \mathrm{p}^{5} 3 \mathrm{~s}(1 / 2,1 / 2)_{1}$ et inférieure à $1 / 1000$ d'angström sur la transition $2 \mathrm{p}^{6}{ }^{1} \mathrm{~S}_{0}-2 \mathrm{p}^{5} 3 \mathrm{~s}(3 / 2,1 / 2)_{2}$.

Les figures 3,4 et 5 , représentant les variations des énergies des configurations $2 \mathrm{p}^{5} 31$ le long de la séquence isoélectronique entre $\mathrm{Al}$ IV et $\mathrm{Fe}$ XVII, permettent de prédire la position de ces niveaux pour les éléments voisins. Les valeurs expérimentales sont de Kaufman et al. pour Al IV [20], de Brillet pour Si V [21], de Eidelsberg et Artru pour P VI [22], de Kononov et al. pour S VII [23], de Jupén pour Cl VIII [24] et des articles cités précédemment [1, 14, $15,2]$ pour les éléments plus lourds. La variation du niveau $2 p^{5} 3 p^{1} S_{0}$ reste encore assez mal déterminée et nécessiterait des mesures complémentaires.

\section{Durées de vie.}

Nous avons pu mesurer la durée de vie des niveaux $2 p^{5} 3 d{ }^{3} F_{4}$ et $2 p^{5} 3 p{ }^{3} D_{3}$ par analyse des déclins radiatifs des transitions intenses $2 p^{5} 3 p^{3} D_{3}-2 p^{5} 3 d^{3} F_{4}$ $(321,28 \AA)$ et $2 p^{5} 3 s(3 / 2,1 / 2)_{2}-2 p^{5} 3 p^{3} D_{3}(405,11 \AA)$.

La courbe de décroissance de la transition située à $321,28 \AA$ permet d'extraire après soustraction du bruit de fond, trois constantes de déclin correspondant à la durée de vie du niveau $2 \mathrm{p}^{5} 3 \mathrm{~d}^{3} \mathrm{~F}_{4} \tau=0,106 \mathrm{~ns}$ et à deux composantes de faibles intensités de valeurs $\tau^{\prime}=0,35 \mathrm{~ns}$ et $\tau^{\prime \prime}=1,24 \mathrm{~ns}$ dues à la contribution des cascades. La valeur expérimentale trouvée est proche de celle calculée par Bureeva et Safranova [16] ( $\tau=0,129 \mathrm{~ns})$, mais comme on pouvait déjà le remarquer dans le cas de l'argon [1] plus petite que la valeur théorique. Compte tenu de la statistique, l'incertitude sur les durées de vie est estimée à $5 \%$ pour la constante de déclin principale et à $10 \%$ pour les cascades.

L'analyse de la courbe de déclin du niveau $2 p^{5} 3 p^{3} D_{3}$ est beaucoup plus complexe comme pour les éléments plus légers de la séquence. En effet linfluence de la cascade provenant du niveau $2 \mathrm{p}^{5} 3 \mathrm{~d}^{3} \mathrm{~F}_{4}$ est très grande, car le peuplement par cascade devient ici du même ordre de grandeur que le peuplement direct. De plus, la constante de déclin de la cascade est plus courte que celle du niveau $2 p^{5} 3 p^{3} D_{3}$ et intervient sous forme de "cascade négative". Dans le cas du chrome, un problème supplémentaire se pose du fait du mélange des deux transitions centrées à $405,1 \AA 2 \mathrm{p}^{5} 3 \mathrm{~s}$ $(1 / 2,1 / 2)_{1}-2 p^{5} 3 p^{3} P_{1}$ et $2 p^{5} 3 s(3 / 2,1 / 2)_{2}-2 p^{5} 3 p^{3} D_{3}$. Pour diminuer au maximum l'influence de la première, nous avons pris garde de nous positionner en longueur d'onde à $405 \AA$ sur le bord du pic et non vers son centroïde. La décomposition de la courbe expérimentale permet d'extraire en plus du bruit de fond et de la durée de vie du niveau $2 p^{5} 3 p^{3} D_{3}(\tau=0,206 \mathrm{~ns})$ deux constantes de déclin $\tau^{\prime}=0,096 \mathrm{~ns}$ et $\tau^{\prime \prime}=1,24 \mathrm{~ns}$ que l'on peut identifier comme correspondant à la contribution très intense du niveau $2 p^{5} 3 d{ }^{3} F_{4}$ et à l'effet moyen des cascades provenant de niveaux plus élevés, compte tenu des résultats trouvés précédemment. La durée de vie mesurée pour le niveau $2 p^{5} 3 p{ }^{3} D_{3}$ est en assez bon accord avec la théorie, la valeur expérimentale étant encadrée par les deux valeurs théoriques données par Crance [19] $\tau=$ $0,187 \mathrm{~ns}$ et par Bureeva et Safronova [16] $\tau=0,222 \mathrm{~ns}$. Malgré la plus grande difficulté d'analyse de ce déclin, la cohérence de la décomposition avec les valeurs trouvées précédemment permet d'estimer l'incertitude sur les durées de vie inférieure ou au plus égale à $10 \%$. 
Tableau III. - Energies des niveaux $2 \mathrm{p}^{5} 31$ de $\mathrm{Cr} \mathrm{XV}$.

[Energies of the $2 \mathrm{p}^{5} 31 \mathrm{Cr} \mathrm{XV}$ levels.]

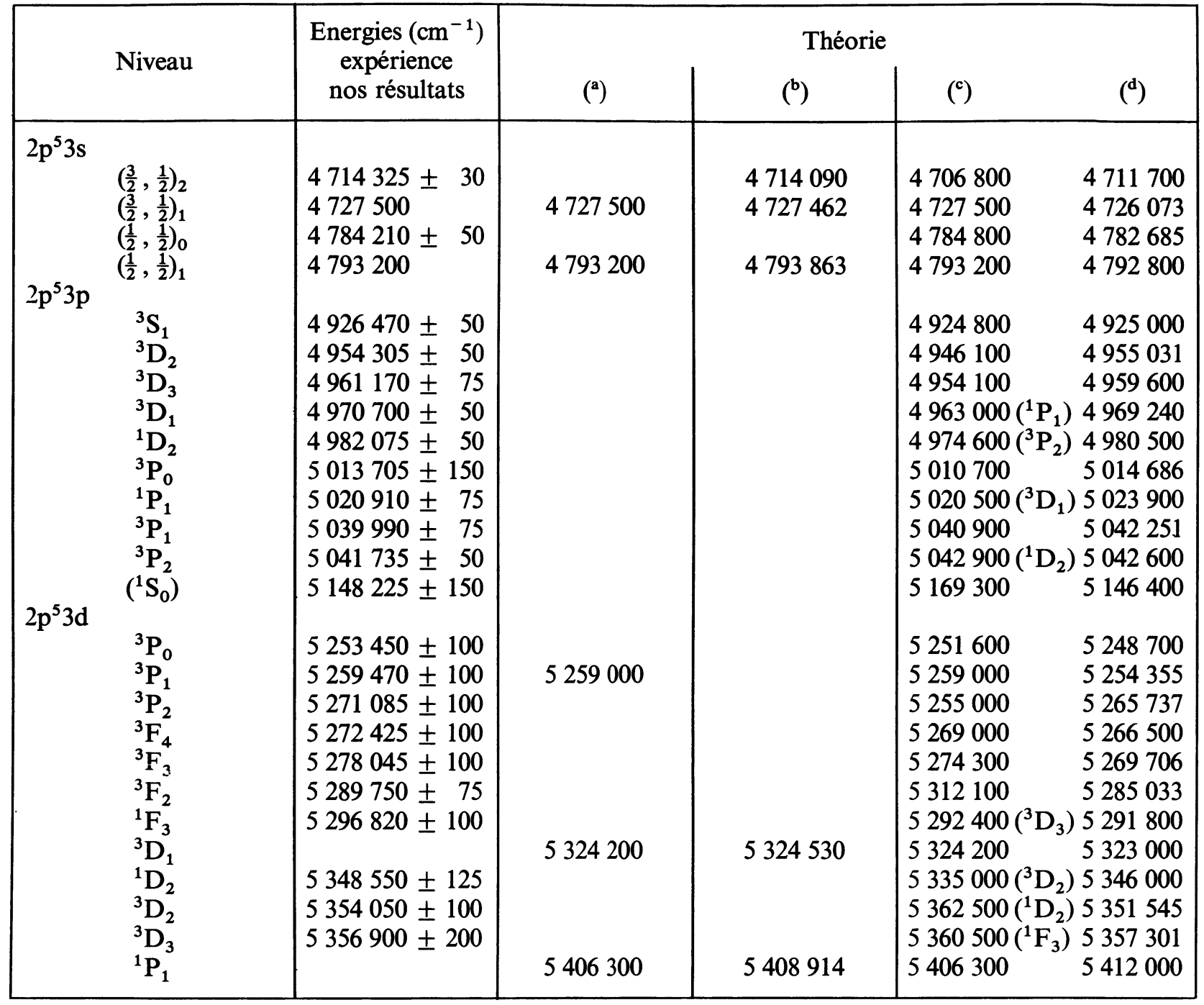

(a) Référence [17]

(b) Déduit de la référence [18]

(c) Référence [19]

(d) Référence [16]

Tableau IV. - Durées de vie des niveaux $2 \mathrm{p}^{5} 3 \mathrm{p}^{3} \mathrm{D}_{3}$ et $2 \mathrm{p}^{5} 3 \mathrm{~d}^{3} \mathrm{~F}_{4}$ de $\mathrm{Cr} \mathrm{XV}$.

[Lifetimes of the $2 p^{5} 3 p^{3} D_{3}$ and $2 p^{5} 3 d^{3} F_{4} C r X V$ levels.]

\begin{tabular}{|c|c|c|c|c|c|c|c|}
\hline \multirow{2}{*}{$\begin{array}{c}\text { Transition } \\
\lambda(\AA)\end{array}$} & \multirow[t]{2}{*}{$\begin{array}{l}\text { Niveau } \\
\text { supérieur }\end{array}$} & \multicolumn{3}{|c|}{$\begin{array}{c}\text { Constantes de déclin } \\
\text { (ns) }\end{array}$} & \multicolumn{3}{|c|}{$\begin{array}{c}\text { Probabilité de transition } \\
\qquad\left(10^{8} \mathrm{~s}^{-1}\right)\end{array}$} \\
\hline & & $\tau$ & $\tau^{\prime}$ & $\tau^{\prime \prime}$ & nous & $\left({ }^{a}\right)$ & $\left({ }^{b}\right)$ \\
\hline 405,01 & $2 p^{5} 3 p^{3} D_{3}$ & $0,206 \pm 0,021$ & $0,096 \pm 0,010$ & $1,24 \pm 0,12$ & 48,5 & 53,35 & 45,04 \\
\hline 321,28 & $2 p^{5} 3 d^{3} F_{4}$ & $0,106 \pm 0,005$ & $0,35 \pm 0,04$ & $1,24 \pm 0,12$ & 94,3 & & 77,5 \\
\hline
\end{tabular}

(a) Référence [19]

(b) Déduit de la référence [16]. 


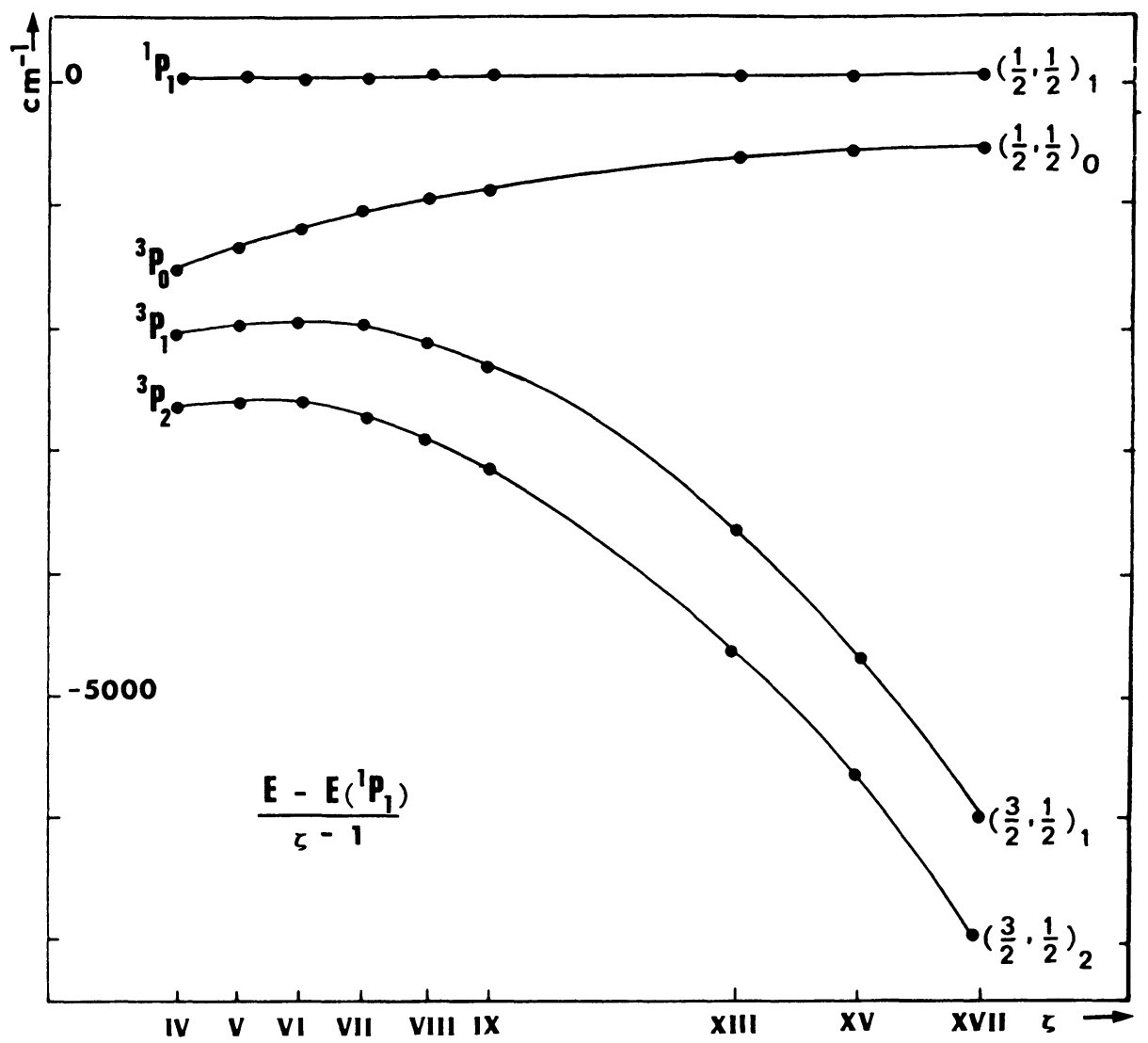

Fig. 3. - Comparaison des configurations $2 \mathrm{p}^{5} 3 \mathrm{~s}$ le long de la séquence isoélectronique. Les points expérimentaux sont tirés des références $[1,2,15,20-24]$ et de ce travail sur $\mathrm{Cr} \mathrm{XV}$.

[Isoelectronic comparison of the $2 p^{5} 3$ s configurations. Experimental dots are from the references $[1,2,15,20-24]$ and from this work for $\mathrm{Cr} \mathrm{XV}$.]

La figure 6 montre la courbe de déclin radiatif du niveau $2 p^{5} 3 p{ }^{3} D_{3}$ et sa décomposition.

Nous résumons dans le tableau IV les résultats trouvés et comparons les probabilités de transition déduites de nos mesures aux valeurs théoriques.

Pour conclure, une dernière remarque peut être faite au sujet des deux transitions $2 \mathrm{p}^{5} 3 \mathrm{p}{ }^{1} \mathrm{~S}_{0} \rightarrow 2 \mathrm{p}^{5} 3 \mathrm{~s}$ $(3 / 2,1 / 2)_{1}$ et $2 p^{5} 3 \mathrm{~s}(1 / 2,1 / 2)_{1}$ : la première, transition d'intercombinaison, est peu intense mais observable. Strictement interdite en couplage LS, elle n'apparaît pas au début de la séquence quand les effets relativistes sont faibles. Par contre, Dere [25] a identifié cette transition dans Fe XVII émis au cours d'éruptions solaires. Kastner [26] suggérait que cette transition qui n'avait pas été observée dans les sources conventionnelles devrait probablement l'être dans des sources diluées comme les Tokamaks ou les sources « beam-foil ». Nous avons effectivement identifié les raies correspondantes dans nos spectres de Fe XVII et Cr XV mais leurs intensités étaient beaucoup plus faibles que les calculs sur les probabilités de transition $[16,19]$ ne le laissaient prévoir. Ici, on peut estimer le rapport des intensités des deux transitions issues du niveau $2 \mathrm{p}^{5} 3 \mathrm{p}{ }^{1} \mathrm{~S}_{0}: I(237,7 \AA) /$ $I(281,67 \AA)=1 / 5$ alors que Kastner [26] prédit ce rapport de l'ordre 1 pour les éléments de la séquence à partir de $Z=20$. 


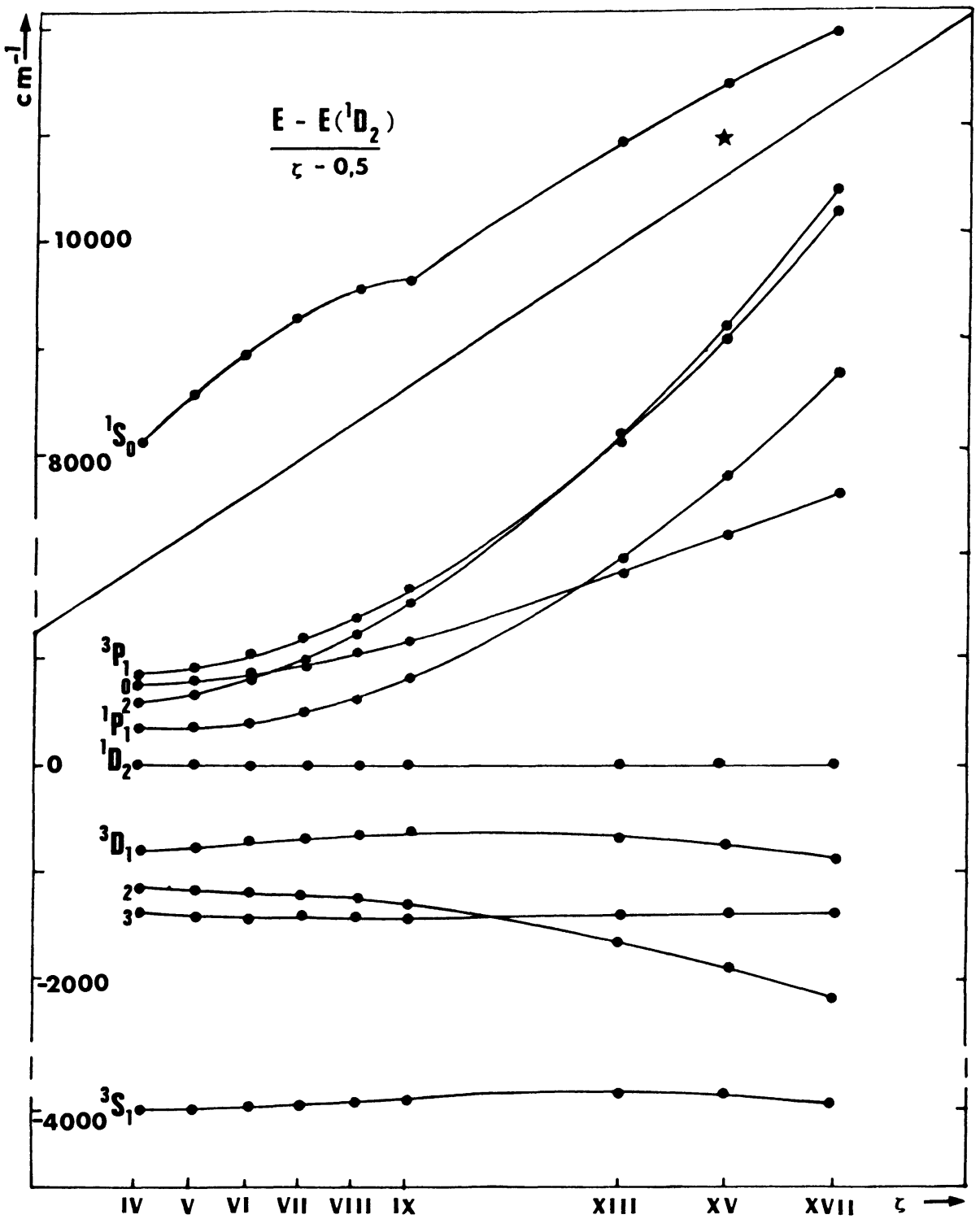

Fig. 4. - Comparaison des configurations $2 \mathrm{p}^{5} 3 \mathrm{p}$ le long de la séquence isoélectronique. Les points expérimentaux sont tirés des références $[1,2,15,20-24]$ et de ce travail sur $\mathrm{Cr} \mathrm{XV}$.

$\star$ montre la position du niveau ${ }^{1} \mathrm{~S}_{0}$ selon l'autre hypothèse mentionnée dans le texte.

[Isoelectronic comparison of the $2 p^{5} 3 p$ configurations. Experimental dots are taken from references $[1,2,15,20-24]$ and from this work for Cr XV.

$\star$ shows the ${ }^{1} \mathrm{~S}_{0}$ position according to the other assumption given in the text.] 


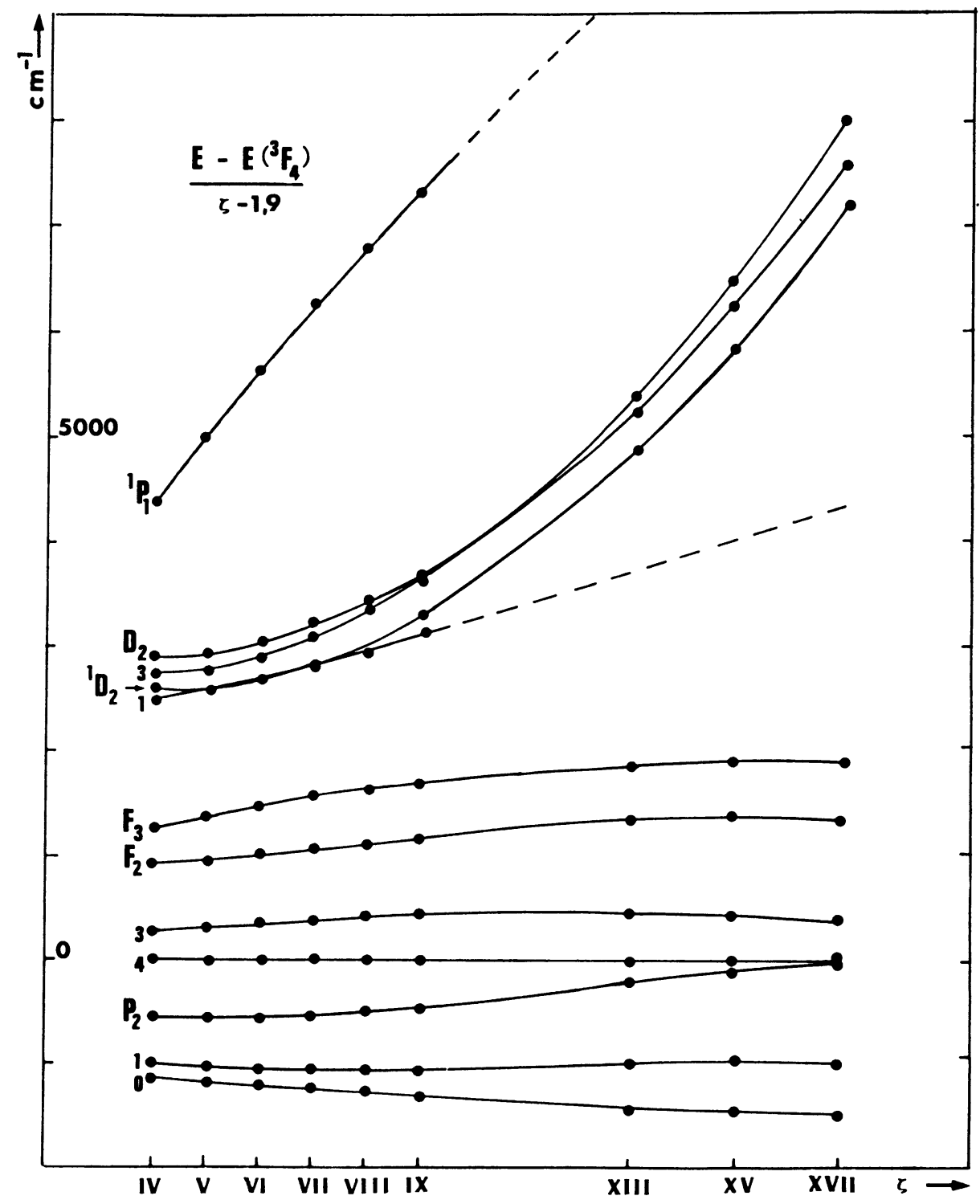

Fig. 5. - Comparaison des configurations $2 \mathrm{p}^{5} 3 \mathrm{~d}$ le long de la séquence isoélectronique. Les points expérimentaux sont tirés des références $[1,2,15,20-24]$ et de ce travail sur $\mathrm{Cr} \mathrm{XV}$.

[Isoelectronic comparison of the $2 \mathrm{p}^{5} 3 \mathrm{~d}$ configurations. Experimental dots are from references [1, 2, 15, 20-24] and from this work for Cr XV.]

\section{Bibliographie}

[1] Buchet-Poulizac, M. C. and Buchet, J. P., Phys. Scripta 27 (1983) 99.

[2] Buchet, J. P., Buchet-Poulizac, M. C., Denis, A., Desesquelles, J., Druetta, M., Martin, S., Grandin, J. P., Husson, X. and LesteVen, I., Phys. Scripta 31 (1985) 364.

[3] Peacock, N. J., Stamp, M. F. and Silver, J. D., Phys. Scripta T 8 (1984) 10.

[4] Breton, C., De Michelis, C., Finkenthal, M. and Mattioli, M., J. Opt. Soc. Am. 69 (1979) 1652.

[5] Fawcett, B. C. and Peacock, N. J., Proc. Phys. Soc. 91 (1967) 973.
[6] Doschex, G. A., Feldman, U., Cowan, R. D. and Cohen, L., Astrophys. J. 118 (1974) 417.

[7] Lawson, K. D. and Peacock, N. J., J. Phys. B At. Mol. Phys. 13 (1980) 3313.

[8] Fawcett, B. C. and Hayes, R. W., Mont. Not. Roy. Astronom. Soc. 170 (1975) 185.

[9] Fawcett, B. C., Atom. Nucl. Dat. Tables 16(1975) 135.

[10] Fawcett, B. C., Atom. Nucl. Dat. Tables 31 (1984) 495.

[11] EdLÉn, B., Phys. Scripta 17 (1978) 565.

[12] Edlén, B., Phys. Scripta 28 (1983) 51.

[13] EdLén, B., Phys. Scripta 30 (1984) 135.

[14] Jupén, C. and Litzén, U., Phys. Scripta 30 (1984) 112. 


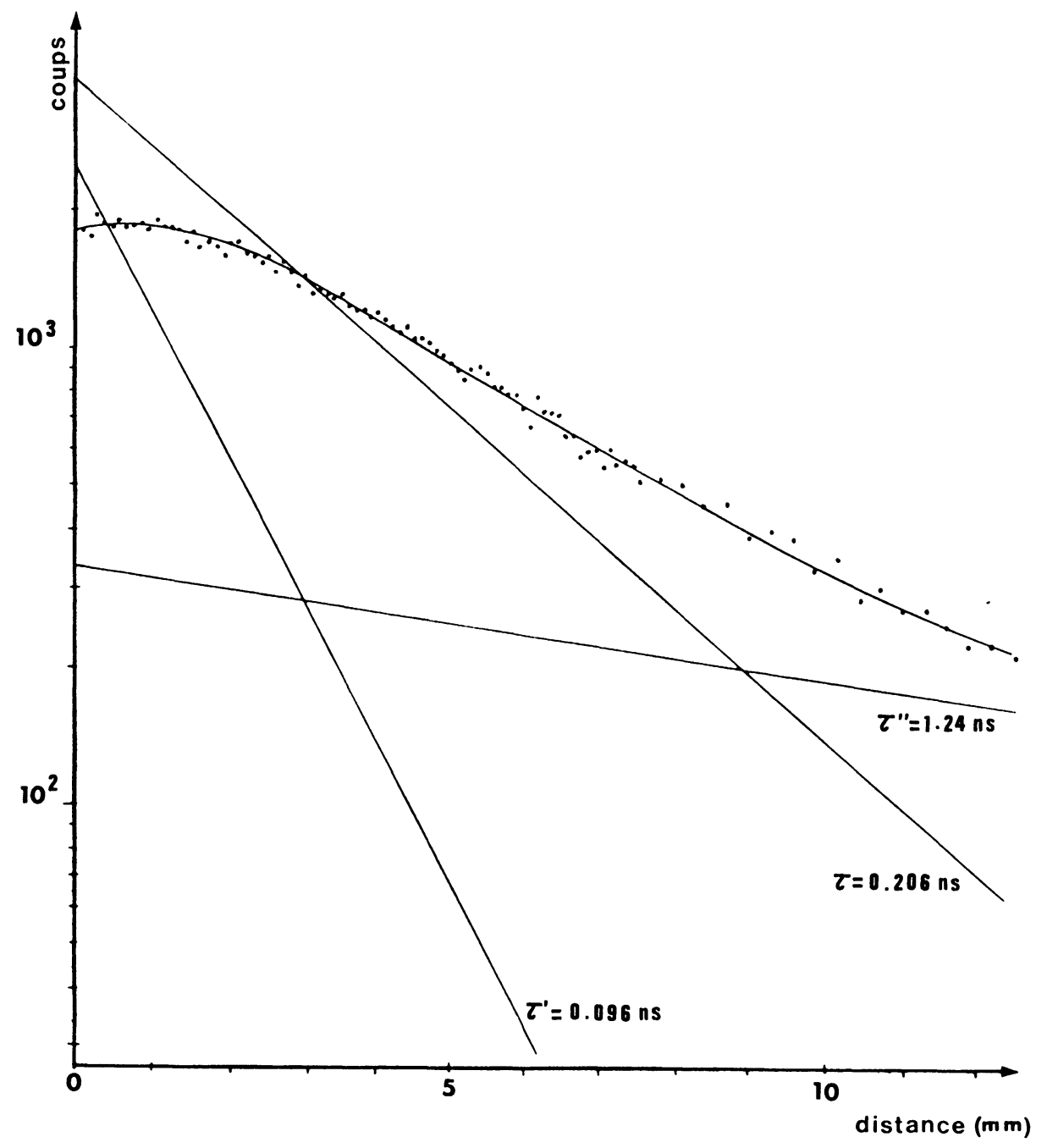

Fig. 6. - Courbe de déclin du niveau $2 \mathrm{p}^{5} 3 \mathrm{p}^{3} \mathrm{D}_{3}$, niveau supérieur de la transition observée à $405,11 \AA$. [Decay curve of the $2 p^{5} 3 p^{3} D_{3}$ level, upper level of the transition at $405.11 \AA$.]

[15] Träbert, E., Z. Phys. A 319 (1984) 25.

[16] Bureeva, L. A. and Safronova, U. I., Phys. Scripta 20 (1979) 81.

[17] Sugar, J. and Corliss, C., J. Phys. Chem. Ref. Data 6 (1977) 317.

[18] Klapisch, M., Bar Shalom, A., Schwob, J. L., Fraenkel, B. S., Breton, C., De Michelis, C., Finkenthal, M. and Mattioli, M., Phys. Lett. 69 A (1978) 34.

[19] Crance, M., Atomic Data 5 (1973) 185.
[20] Kaufman, V., Artru, M. C. and Brillet, W. Ü. L., J. Opt. Soc. Am. 64 (1974) 197.

[21] Brillet, W. Ü. L., Phys. Scripta 13 (1976) 289.

[22] Eidelsberg, M. and Artru, M. C., Phys. Scripta 16 (1977) 109.

[23] Kononov, E. Ya., Kramida, A. E. and Podobedova, L. I., Phys. Scripta 28 (1983) 496.

[24] JUPÉN, C., Nucl. Instrum. Meth. 202 (1982) 25.

[25] Dere, K. P., Astrophys. J. 221 (1979) 1062.

[26] Kastner, S. O., J. Opt. Soc. Am. 70 (1980) 1550. 